\title{
Resource-driven Substructural Defeasible Logic
}

\author{
Francesco Olivieri ${ }^{1}$, Guido Governatori ${ }^{1}$, Matteo Cristani ${ }^{2}$, Nick van Beest ${ }^{1}$ and \\ Silvano Colombo-Tosatto ${ }^{1}$ \\ ${ }^{1}$ Data61, CSIRO, Australia \\ ${ }^{2}$ University of Verona, Italy
}

\begin{abstract}
Linear Logic and Defeasible Logic have been adopted to formalise different features relevant to agents: consumption of resources, and reasoning with exceptions. We propose a framework to combine sub-structural features, corresponding to the consumption of resources, with defeasibility aspects, and we discuss the design choices for the framework.
\end{abstract}

\section{Introduction}

Many different models of agency have been proposed over the years. In some of those understandings, agents are assumed to be rational entities capable to reason about the environment in which they are situated, and to deliberate about what actions to take to achieve some particular goals.

Many logic-based approaches have been proposed to account for the rational behaviour of an agent. For example, in the well known BDI architecture (and architectures inspired by it), agents first deliberate about the goals to achieve and, based on such goals, they select the plans to implement from their plan libraries. Finally, during or after the execution of the plans, the agents receive feedback from the environment, which can trigger the so-called reconsideration: the activity to determine whether the intended goals are still achievable with the selected plan and the current state of execution.

Most of the logic-based approaches take an idealised representation: the agents have unlimited reasoning power, complete knowledge of the environment and their capabilities, and unlimited resources. Over the years, a few approaches (using different logics) have been advanced to overcome some of these ideal (unrealistic) assumptions.

In [10 14 15], the authors propose the use of Linear Logic to model the notion of resource utilisation, and to generate which plans the agent adopts to achieve its goals. In the same spirit, [8]7] address the problem of agents being able to take decisions from partial, incomplete, and possibly inconsistent knowledge bases, using (extensions of) Defeasible Logic (a computational and proof theoretic approach) to non-monotonic reasoning and reasoning with exceptions. While these last two approaches seem very far apart, they are both based on proof theory (where the key notion is on the idea of (logical) derivation), and both logics (for different reasons and different techniques) have been used for modelling business processes [9|17|2|16 13 12 |

Formally, a business process can be understood as a compact representation of a set of traces, where a trace is a sequence of tasks. A business process is hence equivalent to a set of plans with possible choices. The idea behind the work mentioned above is to allow agents to use their deliberation phase to determine the business processes (instead of the plans) to execute. 
In this paper, we discuss some design choices (and offer some results) about the combination of linear logic (or more in general, sub-structural logic) and a computationally oriented non-monotonic formalism. To the best of our knowledge, this is the first investigation in this area, but it combines two approaches that have proved useful for modelling some aspects of agency.

We expect this work to be foundational for further research in modelling agents and the way agents create their plans during the deliberation phase, taking into account the utilisation of resources and possible exceptions (or partial knowledge of the environment). Hereafter, we focus on introducing the key logical aspect to be examined in the paper.

Logic is often described as the "art" of reasoning, or in other terms, its subject matter is how to derive conclusions from given premises. Under this prospective we can distinguish rules (or sequents, or instances of a consequence relation) and inference (or derivation) rules. A rule specifies that some consequences follow from some premises, while a derivation rule provides a recipe to determine the valid steps in a proof or derivation. A classical example of a derivation rule is Modus Ponens (i.e., from ' $\alpha \rightarrow \beta$ ' and $\alpha$ to derive $\beta$ ). A rule can be understood as a pair

$$
\Gamma \vdash \Theta
$$

where $\Gamma$ and $\Theta$ are collections of formulas in an underling language. In Classical Logic, $\Gamma$ and $\Theta$ are sets of formulas and in Intuitionistic Logic $\Theta$ is a singleton. Thus the rules

$$
\alpha, \beta \vdash \gamma, \delta \quad \text { and } \quad \beta, \alpha \vdash \delta, \gamma
$$

are the same rule. Where "," in the antecedent $\Gamma$ is understood as conjunction and disjunction in the consequent $\Theta$. In substructural logics (e.g., Lambek Calculus, and the family of Linear Logics), $\Gamma$ and $\Theta$ are assumed to have an internal structure, and they are considered as multi-sets or sequences. An interpretation of a rule is how to transform the premises in the conclusion. Thus the rule

$$
\alpha, \beta, \alpha \vdash \gamma
$$

can be taken to mean that we need two instances of $\alpha$ with an instance of $\beta$ in between to produce an instance of $\gamma$. Derivation rules, on the other hand, tell us how to combine rules, to obtain new rules. For example, the derivation rule

$$
\frac{\Gamma \vdash \alpha \quad \Theta \vdash \alpha \rightarrow \beta}{\Gamma, \Theta \vdash \alpha, \alpha \rightarrow \beta, \beta}
$$

establishes whether we have a derivation of $\alpha$ from $\Gamma$ and a derivation of $\alpha \rightarrow \beta$ from $\Theta$, then we can combine the $\Gamma$ and $\Theta$, to obtain a derivation, where we have $\alpha$ followed by $\alpha \rightarrow \beta$ and then $\beta$. If the formulas denote activities (or tasks) and resources, then the consequent is a sequence of tasks describing the activities to be done (and the order in which they have to be executed) to produce an outcome (and also, what resources are needed). Thus, we can use the rules to model transformation in a business processes, and derivations as the traces of the process (or the ways in which the process can be executed or the runs of system).

A formalism that properly models processes should feature some key characteristics, and one of the most important ones is to identify which resources are consumed after a task has finished its execution. Consider the notorious vending machine scenario, where the dollar resource is spent to produce the can of cola. Trivially, once we get the cola, the dollar resource is no longer spendable (unless it can be, somehow, replenished). However, the specifications of a process may include thousands of rules to represent at 
their best all the various situations that may occur during the execution of the process itself: situations where the information at hand may be incomplete and, sometimes, even contradictory, and rules encoding possible exceptions. This means that we have to adopt a formalism that is able to represent and reason with exceptions, and partial information.

Defeasible Logic (DL) [11] is a non-monotonic rule based formalism, that has been used to model exceptions and processes. The starting point being that, while rules define a relation between premises and conclusion, DL takes the stance that multiple relations are possible, and it focuses on the "strength" of the relationships. Three relationships are identified: strict rules specifying that every time the antecedent holds the consequent hold; defeasible rules, when the antecedent holds then, normally, the consequent holds; and defeaters when the antecedent holds the opposite of the consequent might not hold. An example of rules with a baseline condition and exception is the scenario the outcome of inserting a dollar coin in a vending machine is that we get a can of cola, unless the machine is out of order, or the machine is switched off. Thus, we can represent this scenario with the rules:

$$
\begin{gathered}
r_{1}: 1 \$ \Rightarrow \text { cola } \\
r_{2}: \text { OutOfOrder } \Rightarrow \neg \text { cola } \\
r_{3}: \text { Off } \Rightarrow \neg \text { cola } .
\end{gathered}
$$

Based on the discussion so far, the motivation of the paper is twofold. From a technical point of view we want to combine, from a logic perspective, the mechanisms of defeasibility with mechanisms from substructural logic (to capture the order of resources, and the consumption of resources). It is clear that the resulting combination of logical machinery could provide a much better formalism for the representation of processes. Accordingly, we will use the point of view of business process modelling to illustrate the technical features we are going to define in the logic (or, to be more specific, for possible variants of substructural defeasible logics).

The remainder of this paper is structured as follows. Section2 2 introduces the reader to the features we want our logics to be equipped with. Subsequently, we provide the formalisation of the logics in Section 3 , and finally, Section 4 concludes our work.

\section{Desired properties}

We dedicate this section to detailing which new features our logics need to implement and, for each of them, to justify their importance with respect to real life problems.

\section{Ordered list of antecedents}

Given the rule ' $r: A, B \Rightarrow C$ ', the order in which we derive $A$ and $B$ is typically irrelevant for the derivation of $C$. As such, $r$ may indistinctively assume the form ' $B, A \Rightarrow C$ '. Consider a login procedure which requires a username and password. Whether we insert one credential before the other does not affect a successful login.

\footnotetext{
${ }^{1} r_{i}$ is the name of rule $i$, symbol $\Rightarrow$ denotes rules meant to derive defeasible conclusions, i.e. conclusions which may be defeated by contrary evidence. As it will be clear in Section 3 . defeasible rules, defeaters, and the superiority relation represent the non-monotonic aspects of our framework.
} 
Nonetheless, sometimes it is meaningful to consider an ordered sequence of atoms in the head of a rule, instead of an unordered set of antecedents. Suppose we have the two activities 'Check Creditworthiness' and 'Approve Loan'. Neither of them depends on the other. However, performing one activity before the other may affect the final result: if we approve the loan before creditworthiness has been checked and approved, then a loan may potentially be provided to someone who is not able to repay.

This allows us to capture the fact that some resources may be independent of each other from the derivational viewpoint (one does not derive the other), but are dependent from a temporal perspective (one must be obtained before the other). Naturally, in the same set/list of antecedents, combinations of unordered and ordered sequences of literals is possible. For instance,

$$
r: A ; B ;(C, D) ; E \Rightarrow F
$$

represents a situation where, in order to obtain $F$, we need to first obtain $A$, then $B$, then either $C$ or $D$ in any order, and lastly, only after both $C$ and $D$ are obtained, we need to obtain $E$. The notation ';' is used as a separator between elements in an ordered sequence, while ',' separates unordered sequences.

\section{Multi-occurrence/repetitions of literals}

From these ideas, it follows that some literals may appear in multiple instances, and that two rules such as

$$
r: A ; A ; B \Rightarrow C \quad \text { and } \quad s: A ; B ; A \Rightarrow C
$$

are semantically different. For instance, rule $r$ may describe a scenario where the order of a product may require two deposit payments followed by a full payment prior to delivery. Regarding $s$, consider that $A$ is now 'Add a tablespoon of ice sugar' and $B$ is 'Stir for 1 minute'. A perfect frosting requires many repetitions of $A$ after $B$ after $A$, for a specific number of repetitions.

Resources consumption

Assume we have two rules,

$$
r: A, B \Rightarrow D \quad \text { and } \quad s: A, C \Rightarrow E .
$$

If we are able to derive $A, B$ and $C$, then $D$ and $E$ are subsequently obtained. Deducing both $D$ and $E$ is a typical problem of resource consumption.

Given the financial state of a customer (i.e., their pay cheque and their monthly spending), a finance approval is sent to the customer for the requested loan. However, that finance approval can only be used once, given the financial situation of that customer. That is, they cannot obtain another loan with the same finance approval. If the customer wants to apply for another loan, they are required to obtain a new finance approval first.

This example indicates that some literals represent resources that are consumed during the derivation process: if they appear in the antecedent of a rule, and such a rule produces its conclusion, then the other rules with the same literals in their antecedent can no longer fire (unless there are multiple occurrences).

Conversely, some resources are not consumed once used. For instance, a policy at a bank may dictate that a customer has to be below 65 years old to be eligible for a mortgage. A similar requirement may hold for a car loan. However, a customer may apply for both a mortgage and a car loan, as neither of these applications invalidate the 
fact that the customer is younger than 65 years old. That is, the information regarding the customers' age is not consumed when used.

The discussion of when a resource has to be considered consumable/non-consumable is outside the scope of this paper. It is a duty of the knowledge engineer to decide whether to tag a resource as consumable, or non-consumable. For the remainder of this paper, we assume all literals to be consumable. The treatment/derivation of nonconsumable literals is the same as in Standard Defeasible Logic (SDL), and thus something well known in the literature of SDL.

\section{Concurrent production}

Symmetrically, we consider two distinct rules having the same conclusion:

$$
r: A \Rightarrow C \quad \text { and } \quad s: B \Rightarrow C .
$$

It now seems reasonable that, if both $A$ and $B$ are derived, then we conclude two instances of $C$ (whereas in classical logics we only know that $C$ holds true). For example, consider a family where it is tradition to have pizza on Friday evening. Last Friday, the parents were unable to communicate with each other during the day, and one baked the pizza while the other bought take-away on the way home.

However, there exists consistent cases where multiple rules for the same literal produce only one instance of the literal (even if they all fire). For example, both a digital or handwritten signature would provide permission to proceed with a request. The same request does not require permission twice: either it is permitted, or it is not.

Resource consumption: A team defeater perspective

Sceptical logics provide a means to decide which conclusion to draw in case of contradicting information. Typically, a superiority relation is given among rules for contrary conclusions: it is possible to derive a conclusion only if there exists a single rule stronger than all the rules for the opposite literal.

Defeasible Logic handles conflicts differently, and the idea here is that of team defeater. We do not look at whether there is a single rule prevailing over all the other rules, but rather whether there exists a team of rules which can jointly defeat the rules for the contrary conclusion. That is, suppose rules $r^{\prime}, r^{\prime \prime}$ and $r^{\prime \prime \prime}$ all conclude $P$, whilst $s^{\prime}$ and $s^{\prime \prime}$ are for $\neg P$. If $r^{\prime}>s^{\prime}$ and $r^{\prime \prime}>s^{\prime \prime}$, then the team defeater made of $\left\{r^{\prime}, r^{\prime \prime}\right\}$ is sufficient to prove $P$.

The focus remains on resource consumption and production. As such, the questions we need to answer are, again, which resources are consumed, and how many instances of the conclusion are derived. We start by distinguishing the two scenarios where: (a) neither of the teams prevail, (b) one team wins. Consider

$$
r^{\prime}: A \Rightarrow P, \quad r^{\prime \prime}: B \Rightarrow P, \quad r^{\prime \prime \prime}: C \Rightarrow P, \quad s^{\prime}: D \Rightarrow \neg P, \quad s^{\prime \prime}: E \Rightarrow \neg P .
$$

In case (a), e.g., when no superiority is given, we cannot conclude for either conclusion. Hence, the question is "Will any of the resources be consumed?". In case (b), we assume $r^{\prime}>s^{\prime}$ and $r^{\prime \prime}>s^{\prime \prime}$, and we conclude that $P$. How many instances of $P$ are produced? One solution is to produce three instances of $P$ and, accordingly, $A, B$ and $C$ are all consumed. We can instead consistently assume that we produce $P$ twice, through the two winning rules $r^{\prime \prime}$ and $r^{\prime \prime \prime}$ only, but not via $r^{\prime}$; we thus consume $B$ and $C$, but not $A$.

Lastly, on the perspective of the defeated rules another relevant question is: Are $D$ and $E$ ever consumed? As clear, there is no unique answer. There are consistent 
scenarios where the literals in the defeated rules are consumed, and other cases where they are not. In Section 3, we provide different solutions to cover the various cases.

Consider the process of writing a scientific publication for a conference. If the paper is accepted, the manuscript resource is consumed, since it cannot be submitted again. On the contrary, if the paper is rejected, the manuscript resource is not consumed since it can be submitted again to other venues.

\section{Multiple conclusions and resource preservation}

Consider internet shopping. As soon as we pay for our online order, the bank account balance decreases, the seller's account increases. Both the seller and the web site have the shipping address and, possibly, the credit card number.

The conclusion of a rule is usually a single literal. The above example suggests that a single rule may produce more than one conclusion, which cannot be represented by multiple rules with the same set of antecedents. For example, consider the rules

$$
r: A, B \Rightarrow C \quad \text { and } \quad s: A, B \Rightarrow D .
$$

In a propositional calculus, once the system derives $A$ and $B$, by Modus Ponens, we obtain both $C$ and $D$. However, when we consider resource consumption, then it is clear that only one rule can produce its conclusion, whilst the other cannot. We tackle this problem by allowing rules to have multiple conclusions. Thus, $r$ and $s$ can be merged into the single rule

$$
r^{\prime}: A, B \Rightarrow C, D \text {. }
$$

Similar to our discussion on the ordering of antecedents, we may have any combination of ordered/unordered sequences of conclusions. In the previous example, only after we have provided the credit card credentials, our bank account decreases, whilst we can provide the shipping address before the credit card credentials, or the other way around.

The notion of multiple conclusions, along with the discussion on team defeaters, leads to another problem. Consider the two rules

$$
r: A \Rightarrow B ; C ; D \quad \text { and } \quad s: E \Rightarrow \neg C,
$$

where no superiority is given. Do we conclude that $B$ or $D$ ? Moreover, what happens if now we have ' $r: A \Rightarrow B, C, D$ ' and we establish that $s$ is stronger then $r$ ? Do we conclude that $B$ and $D$ (meaning that only the derivation of $C$ has been blocked by $s$ ), or will the production of $B$ and $D$ be affected also?

\section{Loops}

The importance of being able to properly handle loops is evident: loops play a fundamental role in many real life applications, from business processes to manufacturing. Back to the login procedure, if one of the credentials is wrong, the process loops back to a previous state, for instance, by asking the user to re-enter both credentials.

Naturally, a system is able to properly handle loops when it can handle/recognise the so-called exit conditions, to prevent infinite repetition of the same set of events. For example, after three wrong login attempts, the login procedure may prevent the user from further attempts and require them to undergo a retrieve credential procedure. 


\section{Language and logical formalisation of RSDL}

Before introducing the notation used throughout the remainder of this paper, we will first justify a number of implementation choices. In Section 2, we stated that there are two types of literals: consumable against non-consumable. In addition, we introduced the notion of ordered sequences of literals in the antecedents and conclusions, we stated that any combination of ordered or unordered sequences of literals is (theoretically) possible.

The logics presented here shall only consider: (i) consumable literals, and (ii) either multi-sets or ordered sequences of literals (but not their combination). Our motivation is that adding conditions to the proof tags (labels that describe how a literal can be derived) in order to deal with non-consumable literals and alternating ordered/unordered sequences is a trivial and pedant task. Their formalisation is exactly the same of that in Standard DL (SDL), and thus the process would not add any value (it will be done for the sake of completeness in future work).

Our logics deal with two types of derivations: strict and defeasible. Their underlying meaning is the same as those in SDL. Strict rules derive indisputable conclusions, i.e., conclusions that which are always true. Thus, if two strict rules have opposite conclusions, then the resulting logic is inconsistent. On the contrary, defeasible rules are to derive pieces of information that can be defeated by contrary evidence, like 'Birds typically fly' since we know that 'Penguins are birds that do not fly'. Finally, defeaters are special type of rules whose only purpose is to block contrary evidence. They cannot be used to directly derive conclusions, but only to prevent other rules to conclude.

We have now passed through the conceptual basis of our logics and can move forward to introduce the language of Resource-driven Substructural Defeasible Logic (RSDL). We will use greek letters to denote propositional atoms, while roman letters $r$, $s, t$ are reserved to denote rule labels. In addition, $l$ and $c$ are reserved to denote lines and columns in a proof, while other roman letters are typically used as subscripts to denote the cardinality of sets/sequences; capital $F$ denotes the set of facts, capital $R$ the set of rules, $A(r)$ the list of antecedents, and $C(r)$ the set/list of conclusions of rule $r$.

PROP is the set of propositional atoms, the set Lit $=\operatorname{PROP} \cup\{\neg \varphi \mid \varphi \in \operatorname{PROP}\}$ denotes the set of literals. The complement of a literal $\varphi$ is denoted by $\sim \psi$; if $\psi$ is a positive literal $\varphi$, then $\neg \psi$ is $\neg \varphi$, and if $\psi$ is a negative literal $\neg \varphi$, then $\sim \psi$ is $\varphi$.

We adopt the standard DL definitions of strict rules, defeasible rules, and defeaters.

Definition 1. Let Lab be a set of arbitrary labels. Every rule is of the type $r: A(r) \hookrightarrow$ $C(r)$, where

1. $r \in \mathrm{Lab}$ is a unique name.

2. $A(r)=\alpha_{1}, \ldots, \alpha_{n}$, the antecedent, or body, of the rule is a list of literals.

3. An arrow $\hookrightarrow \in\{\rightarrow, \Rightarrow, \rightsquigarrow\}$ denoting a strict rule, a defeasible rule, and a defeater, respectively;

4. $C(r)$ is the consequent, or head, of the rule. For the head, we consider three options:

(a) The head is a single literal $\varphi$;

(b) The head is a list $\varphi_{1}, \ldots, \varphi_{m}$ (to be understood as a multi-set);

(c) The head is a list $\varphi_{1} ; \ldots ; \varphi_{m}$ (to be understood as a ordered list).

With abuse of notation, we will often refer to $\varphi_{1}, \ldots, \varphi_{m}$ as a set, and overload standard set theoretic notation. Given a set of rules $R$, and a rule $r: A(r) \hookrightarrow C(r)$ we 
use the following abbreviations for specific subsets of rules: (i) $R_{S}$ is the subset of strict rules, (ii) $R_{s d}$ is the set of strict and defeasible rules, (iii) $R[\varphi ; i]$ is the set of rules where $\varphi$ appears at index $i$ in the consequent where the consequent is a list, (iv) $R[\varphi, i]$ when the consequent is a set containing $\varphi$.

Definition 2. A resource-driven substructural defeasible (rsd) theory is a tuple $(F, R, \succ$ ) where: (i) $F \subseteq$ Lit are pieces of information denoting the (consumable) resources available at the beginning of the computation. This differs strikingly from SDL, where i) they denote always-true statements; (ii) $R$ is the set of rules; (iii) $\succ$, the superiority relation, is a binary relation over $R$.

A theory is finite if the set of facts and rules are finite. In SDL, a proof $P$ of length $n$ is a finite sequence $P(1), \ldots, P(n)$ of tagged literals of the type $\pm \Delta \varphi$ and $\pm \partial \varphi$. The idea is that, at every step of the derivation, a literal is either proven or disproven.

In our logic, we must be able to derive multiple conclusions in a single derivation step, and hence we require a mechanism to determine when premises have been used to derive conclusion. Accordingly, we modify the definition of proof to be a matrix.

Definition 3. A proof $P$ in $R S D L$ is $P(l, c)$ a finite matrix $P(1,1), \ldots, P(l, c)$ of tagged literals of the type $\pm \Delta \varphi, \pm \partial \varphi,+\sigma \varphi,+\Delta \varphi^{\checkmark}$ and $+\partial \varphi^{\checkmark}$.

We assume that facts are simultaneously true at the beginning of the computation. Notation $+\# \varphi^{\checkmark}, \# \in\{\Delta, \partial\}$, denotes the fact that $\varphi$ has been consumed. The distinctive notation for when a literal is proven and when it is consumed will play a key role to determine which rules are applicable.

The tagged literal $\pm \Delta \varphi$ means that $\varphi$ is strictly proved/refuted in $D$, and, symmetrically, $\pm \partial \varphi$ means that $\varphi$ is defeasibly proven/refuted. The set of positive and negative conclusions is called extension.

In SDL, given a set of facts, a set of rules and a superiority relation, the extension is unique. It is clear that this is not the case when resource consumption and ordered sequences are to be taken into account: depending on the order in which the rules are applied, (rather) different extensions can be obtained. In sub-structural defeasible logic every distinct derivation corresponds to an extension.

In SDL, derivations are based on the notions of a rule being applicable or discarded. Briefly, a rule is applicable when every literal in the antecedent has been proven at a previous step. We report hereafter a standard defeasible proof tag.

If $P(n+1)=+\partial \varphi$ then

(1) $\exists r \in R_{s d}[\varphi]: r$ is applicable and

(2) $\forall s \in R[\sim \varphi]$ either

(2.1) $s$ is discarded or

(2.2) $\exists t \in R[\varphi]: t$ is applicable and $t \succ s$.

A literal is defeasibly proven when there exists an applicable rule for such a conclusion and all the rules of the opposite are either discarded, or defeated by stronger rules. (Strict derivations only differ in that, when a rule is applicable, we do not care about contrary evidence, and the rule will always produce its conclusion nonetheless. As such, the consistency of the logics depends only on the strict part of the logics.)

As for SDL, we obtain variants of the logic by providing different definitions of being applicable and discarded. More specifically, for RSDL, definitions of applicability 
and discardability need to take into account (i) the number of times a literals appear in the body of a rule, (ii) how many times they have been derived 2 , (iii) the order in which the literals occur in the body of a rule and in a derivation. In addition, we have to extend the structure of the proof conditions to include mechanisms or conditions to determine when literals/resources have been used to derive new literals/resources.

We shall proceed incrementally. First, we provide definitions for multi-sets. We then provide definitions for sequences. In both cases, we consider rules with a single literal for conclusion. Consequently, we end with the definitions to describe multiple conclusions.

Definition 4. A rule $r$ is \#-applicable, $\# \in\{\Delta, \partial, \sigma\}$, at $P(l+1, c+1)$ iff $\forall \alpha_{i} \in A(r)$ then $+\# \alpha_{i} \in P[(1,1) . .(l, c)]$. Moreover, we say that $r$ is \#-consumable iff $r$ is \#-applicable and $\exists l^{\prime} \leq l$ such that $P\left(l^{\prime}, c\right)=+\# \alpha_{i}$.

A rule is consumable if it is applicable and, for every literal in its antecedent, there is an unused occurrence. (This can be done by checking the previous derivation step $c$.) Discardability is obtained by applying the principle of the strong negation to the definition of applicability.

Definition 5. A rule $r$ is \#-discarded, $\# \in\{\Delta, \partial\}$, at $P(l+1, c+1)$ iff $\exists \alpha_{i} \in A(r)$ such that $-\# \alpha_{i} \in P[(1,1) . .(l, c)]$. Moreover, we say that $r$ is \#-non-consumable iff either $r$ is discarded, or $\forall l^{\prime} \leq l, P\left(l^{\prime}, c\right) \neq+\partial \alpha_{i}$.

Lastly, we define the conditions describing when a literal is consumed.

Definition 6. Given rule $r$, a literal $\alpha \in A(r)$ is \#-consumed, $\# \in\{\Delta, \partial\}$, at $P(l+1, c+$ 1), iff

1. $\exists l^{\prime} \leq l$ such that $P\left(l^{\prime}, c\right)=+\# \alpha$, and

2. $P\left(l^{\prime}, c+1\right)=+\# \alpha^{\checkmark}$.

The following example illustrates how we use $+\partial \varphi^{\checkmark}$ within the resource consumption mechanism.

Example 1. Consider $D=(\{\alpha\}, R, \emptyset)$, where

$$
R=\left\{r_{0}: \alpha \Rightarrow \beta, \quad r_{1}: \beta \Rightarrow \varphi, \quad r_{2}: \beta \rightarrow \psi\right\}
$$

and (one of) the corresponding proof table(s):

\begin{tabular}{c|ccc}
$P$ & 1 & 2 & 3 \\
\hline 1 & $+\Delta \alpha+\Delta \alpha^{\checkmark}$ & $+\Delta \alpha^{\checkmark}$ \\
2 & & $+\partial \beta$ & $+\partial \beta^{\checkmark}$ \\
3 & & & $+\partial \varphi$
\end{tabular}

Naturally, two mutually exclusive extensions are possible, based on whether $+\partial \beta$ is used by $r_{1}$ to derive $+\partial \varphi$, or by $r_{2}$ to derive $+\partial \psi$. Table 1 shows the former case. At $P(1,1)$ we obtain $+\Delta \alpha$, instance that is consumed in deriving $+\partial \beta$ at $P(2,2)$. Thus, $P(1,2)=+\Delta \alpha^{\checkmark}$. Symmetric situation for activating $r_{1}$ at the third derivation step, which results in $P(2,3)=+\partial \beta^{\checkmark}$ and $P(3,3)=+\partial \varphi$. Now, at the fourth derivation step, $r_{2}$ is applicable but non-consumable, and hence we cannot derive $+\partial \psi$.

\footnotetext{
${ }^{2}$ Trivially, e.g., if literal $\alpha$ has been derived twice, but it appears in the antecedent of three rules, only two of such rules can produce their conclusions.
} 


\section{Proof tags for multi-sets in the antecedent and single conclusion}

We now present the proof tags, and we begin with: (1) the antecedent is a multi-set, (2) single literal in the conclusion. $+\Delta$ describes positive definitive (strict) derivations.

$+\Delta$ : If $P(l+1, c+1)=+\Delta \varphi$ then

(1) $\varphi \in F$, or

(2) (1) $\exists r \in R_{S}[\varphi]$ such that

(2) $r$ is $\Delta$-consumable and

(3) $\forall \alpha_{j} \in A(r), \alpha_{j}$ is $\Delta$-consumed.

Literal $q$ is definitely provable if it either is a fact, or there is a strict, applicable rule for $\varphi$, whose antecedent literals can be consumed. Condition (2) actually consumes the literals by replacing $+\Delta \alpha_{j}$ with $+\Delta \alpha_{j}^{\checkmark}$. Proof tag $-\Delta$ is as follows:

$-\Delta$ : If $P(l+1, c+1)=-\Delta \varphi$ then

(1) $\varphi \notin F$ and

(2) $\forall r \in R_{S}[\varphi], r$ is $\Delta$-non-consumable.

Literal $q$ is definitely refuted if $\varphi$ is not a fact, and every rule for $\varphi$ is non-consumable. We can now turn our attention to the definition of the proof tags for defeasible conclusions. In particular, we provide proof conditions (corresponding to inference rules) for three types of conclusions: $+\partial \varphi$ meaning that the current derivation $P$ proves $\varphi ;-\partial \varphi$ meaning that the current derivation $P$ prevents the proof of $\varphi$, or in other terms that $\varphi$ is refuted; and $+\sigma \varphi$ whose intuitive reading is that the $\varphi$ would be derivable in the current proof if more (appropriate) resources are would be available. Alternatively, for $+\sigma \varphi$ indicates that there are applicable rules for $\varphi$, but the resources for such rules have been used to derive other conclusions.

$+\partial:$ If $P(l+1, c+1)=+\partial \varphi$, then

(1) $+\Delta \varphi \in P(l, c)$ or

(2) (1) $-\Delta \sim \varphi \in P(l, c)$ and

(2) $\exists r \in R_{s d}[\varphi] \partial$-consumable and

(3) $\forall s \in R[\sim \varphi]$ either

(1) $s$ is $\partial$-discarded, or

(2) $\exists t \in R[\varphi] \partial$-consumable, $t \succ s$, and

(3) if $\exists w \in R[\sim \varphi] \partial$-applicable, $t \succ w$, then

(1) $\forall \alpha_{j} \in A(t), \alpha_{j}$ is $\partial$-consumed, otherwise

(2) $\forall \alpha_{k} \in A(r), \alpha_{k}$ is $\partial$-consumed.

Condition (1) allows us to inherit a defeasible derivation from a definite derivation. Condition (2.1) ensures that the logic is sound (i.e., that it is not possible to derive $\varphi$ and its negation as provable defeasible conclusions unless they are derivable from the strict part only. Condition (2.2) requires that there is rules that is triggered by previously proved literals that have not been used to trigger other conclusions. Clause (2.3.1) is the standard one of defeasible logic, meaning that to rebut an attacking argument, we can show that some of the premises of the argument/rule have been refuted. The second method to rebut the attacking arguments (rules for $\sim \varphi$ ) is to show that they are defeated, i.e., weaker than applicable rules for the conclusion we want to prove, similarly the 
antecedents of such rules must not have been used for other conclusions, clause (2.3.2). The final part is the mechanism to determine which resources are consumed by the derivation of $\varphi$. If there are no applicable rules for $\sim \varphi$ the resources are taken for the rule proposed as an argument, that is rule $r$ (2.3.3.2); if there are counter-argument, the resources are taken from each rule rebutting a counter-argument, for each possible applicable counterargument (2.3.3.1). Note, that in this way we capture the idea of team defeat (see Section 2

For $-\partial$ we use the strategy similar to that used in [1] to provide proof condition for the ambiguity propagating variant of SDL, that is, we make it easier to attack a rule (2.2.2). Also, for the derivation of literals tagged with $-\partial$ does not require the consumption of resources. Resources are consumed only when we positively derive literals.

$-\partial$ : If $P(l+1, c+1)=-\partial \varphi$, then

(1) $-\Delta \varphi \in P[l, c]$ and

(2)(1) $+\Delta \sim \varphi \in P[l, c]$ or

(2) $\forall r \in R_{s d}[\varphi]$ either

(1) $r$ is $\partial$-discarded or

(2) $\exists s \in R[\sim \varphi]$ such that

(1) $s$ is $\sigma$-applicable and

(2) $\forall t \in R[\varphi]$ either

(1) $t$ is $\partial$-discarded or (2) not $t \succ s$.

The idea behind $+\sigma$ is that there are rules applicable for the consequent, irrespective whether the premises have been used or not. However, we have to check that the rule is not defeated by an applicable rule for the opposite (2.2).

$+\sigma$ : If $P(l+1, c+1)=+\sigma \varphi$ then

(1) $\Delta \varphi \in P[l, c]$ or

(2) $\exists r \in R_{s d}[\varphi]$ such that

(1) $r$ is $\sigma$-applicable and

(2) $\forall s \in R[\sim \varphi]$ either

(1) $s$ is $\partial$-discarded or (2) not $s \succ r$.

Example 2. Consider $D=\left(\{\alpha, \beta, \gamma\}, R, \succ=\left\{\left(r_{2}, r_{3}\right)\right\}\right)$, where

$$
R=\left\{r_{0}: \alpha \Rightarrow \varphi, \quad r_{1}: \alpha \Rightarrow \psi, \quad r_{2}: \beta \Rightarrow \varphi, \quad r_{3}: \gamma \Rightarrow \sim \varphi\right\},
$$

and the corresponding proof table:

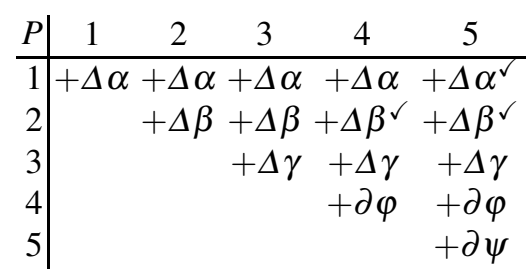

Assume that, at the fourth derivation step, $r_{0}$ is taken into consideration; $r_{0}$ is actually consumable, but so is $r_{3}$, and no superiority is given between $r_{0}$ and $r_{3}$. There actually exists a consumable rule stronger than $r_{3}, r_{2}$. Accordingly, the team defeater allows us to prove $+\partial \varphi$, and only $\beta$ is consumed in this process. This implies that $\alpha$ is still available, and can be used at the fifth derivation step to produce $+\partial \psi$, via $r_{1}$. 


\section{Proof tags for sequences in the antecedent and single conclusion}

We can now move forward and consider sequences in the antecedent. Naturally, definitions of being applicable, discarded, and consumable must be revised.

A rule is sequence applicable when the derivation order reflects the order in which the literals appear in the antecedent, i.e., for every two literals in the antecedent, say $\alpha$ and $\beta$, such that $\alpha$ appears before $\beta$, there exists a derivation of $\alpha$ before every derivation (for that occurrence $\sqrt{3}$ ) of $\beta$.

Definition 7. A rule $r \in R[\varphi]$ is \#-sequence applicable, \# $\in\{\Delta, \partial\}$, at $P(l+1, c+1)$ iff for all $\alpha_{i} \in A(r)$ :

1. there exists $c_{i} \leq c$ such that $P\left(l_{i}, c_{i}\right)=+\# \alpha_{i}, l_{i} \leq l$, and

2. for all $\alpha_{j} \in A(r)$ such that $i<j$, then

3. for all $c_{j} \leq c$ such that $P\left(l_{j}, c_{j}\right)=+\# \alpha_{j}, l_{j} \leq l$ then $l_{i}<l_{j}$ and $c_{i}<c_{j}$.

We say that $r$ is \#-sequence consumable iff is \#-applicable and 4. $P\left(l_{i}, c\right)=+\# \alpha_{i}$.

A rule is sequence discarded when there exists a literal in the antecedent, which has been previously disproven, or there are two proven literals in the antecedent, say $\alpha$ and $\beta$, such that $\alpha$ appears before $\beta$, and one proof for $\beta$ is before every proof for $\alpha$.

Definition 8. A rule $r \in R[\varphi]$ is \#-sequence discarded, \# $\in\{\Delta, \partial\}$, at $P(l+1, c+1)$ iff for there exists $\alpha_{i} \in A(r)$ such that either

1. $-\# \alpha_{i} \in P(l-1, c-1)$, or

2. for all $c_{i} \leq c$ such that $P\left(l_{j}, c_{i}\right)=+\# \alpha_{i}, l_{i} \leq l$, then

- there exists $\alpha_{j} \in A(r)$, with $i<j$, such that

- there exists $c_{j} \leq c$ such that $P\left(l_{j}, c_{j}\right)=+\# \alpha_{j}, l_{j} \leq l$, and $c_{i}>c_{j}, l_{i}>l_{j}$.

The definition of a literal being \#-consumed remains the same as before. The proof tags for strict and defeasible conclusions with (i) sequences in the antecedent, and (ii) a single conclusion can be obtained by simply replacing

- \#-applicable with \#-sequence applicable;

- \#-consumable with \#-sequence consumable;

- \#-discarded with \#-sequence discarded.

Example 3. Consider $D=(\{\gamma, \varepsilon, \zeta\}, R, \succ=\emptyset)$, where $R=\left\{r_{0}: \alpha, \beta, \alpha \Rightarrow \varphi, \quad r_{1}: \gamma \Rightarrow\right.$ $\left.\alpha, \quad r_{2}: \varepsilon \Rightarrow \alpha, \quad r_{3}: \varepsilon \Rightarrow \beta\right\}$.

Assume the rules are activated in this order: first $r_{1}$, then $r_{2}$, last $r_{3}$. Thus, $P(4,4)=$ $+\partial \alpha, P(5,5)=+\partial \alpha$, and $P(6,6)=+\partial \beta$. The derivation order between $\beta$ and the second occurrence of $\alpha$ has not been complied with, and $r_{0}$ is sequence discarded. Same if the order is ' $r_{3}, r_{1}, r_{2}$ ', whilst ' $r_{2}, r_{3}, r_{1}$ ' is a legit order to let $r_{0}$ be sequence applicable.

\section{Proof tags for sequences in both the antecedent and conclusion}

Even when we consider sequences in the consequent, a literal's strict provability or refutability depends only upon whether the strict rule (where the literal occurs) is sequence consumable or not. As such, given a strict rule $r \in R_{S}[\varphi ; j]$, still $\varphi$ 's strict provability/refutability depends only upon whether $r$ is strictly sequence consumable or not. However, now we also have to verify that, if $r \in R_{s}[\psi ; j-1]$, we prove $\varphi$ immediately after $\psi$. The resulting new formalisation of $+\Delta$ is as follows:

\footnotetext{
${ }^{3}$ In order to handle situations like $A(r)=\{\alpha ; \beta ; \alpha ; \beta\}$ (as illustrated in Example 3).
} 
$+\Delta$ : If $P(l+1, c+1)=+\Delta \varphi$ then

(1) $\varphi \in F$, or

(2) (1) $\exists r \in R_{s}[\varphi ; j]$ such that

(2) $r$ is $\Delta$-sequence-consumable,

(3) $r \in R_{s}[\psi ; j-1]$ and $P(l+i-1, c+1)=+\Delta \psi$,

(4) $\forall \alpha_{j} \in A(r), \alpha_{j}$ is $\Delta$-consumed.

The positive, defeasible proof tag is as follows.

$+\partial$ : If $P(l+i, c+1)=+\partial \varphi$, then

(1) $+\Delta \varphi \in P(l, c)$ or

(2) (1) $-\Delta \sim \varphi \in P(l, c)$ and

(2) (1) $\exists r \in R_{s d}[\varphi ; j] \partial$-sequence-consumable and

(2) $\exists r \in R[\psi ; j-1]$ and

(3) $P(l+i-1, c+1)=+\partial \psi$, and

(3) $\forall s \in R[\sim \varphi]$ either

(1) $s$ is $\partial$-sequence-discarded, or

(2) $\exists t \in R[\varphi] \partial$-sequence-consumable, $t \succ s$, and

(3) if $\exists w \in R[\sim \varphi] \partial$-sequence-applicable, $t \succ w$, then

(1) $\forall \alpha_{j} \in A(t), \alpha_{j}$ is $\partial$-consumed, otherwise

(2) $\forall \alpha_{k} \in A(r), \alpha_{k}$ is $\partial$-consumed.

Negative proof tags are trivial to deduce, and therefore omitted.

Example 4. Consider $D=(\{\alpha, \beta\}, R, \succ=\emptyset)$, where

$$
R=\left\{r_{0}: \alpha \Rightarrow \varphi ; \chi ; \psi, \quad r_{1}: \beta \Rightarrow \sim \chi\right\} .
$$

At $P(3,3)$ we prove $+\partial \varphi$, while $P(4,3)=-\partial \chi\left(r_{1}\right.$ is sequence-applicable and $r_{0}$ is not stronger than $\left.r_{1}\right)$. Therefore, $r_{0}$ cannot prove $\psi$.

\section{Proof tags for sequences in the antecedent and multi-sets in the conclusion}

We lastly take into account multi-sets in the conclusion. When considering a 'team defeater fight', two scenarios are possible. In the former, we draw a conclusion only if there is a winning team defeater for each literal in the conclusion. In the latter, we limit the comparison on the individual literal (and thus the latter solution is less strict than the former).

Strict provability does not change with respect to the one described in the previous section, and is therefore omitted.

$+\partial:$ If $P(l+i, c+1)=+\partial \varphi$, then

(1) $+\Delta \varphi \in P(l, c)$ or

(2) (1) $-\Delta \sim \varphi \in P(l, c)$ and

(2) $\exists r \in R_{s d}[\varphi, j] \partial$-sequence-consumable and

(3) $\forall s \in R[\sim \psi]$ such that $\psi \in C(r)$ either

(1) $s$ is $\partial$-sequence-discarded, or

(2) $\exists t \in R[\psi] \partial$-sequence-consumable, $t \succ s$, and

(3) if $\exists w \in R[\sim \varphi] \partial$-sequence-applicable, $t \succ w$, then

(1) $\forall \alpha_{j} \in A(t), \alpha_{j}$ is $\partial$-consumed, otherwise

(2) $\forall \alpha_{k} \in A(r), \alpha_{k}$ is $\partial$-consumed. 
Consider $D$ of Example 4, where this time $C(r)=\{\varphi, \chi, \psi\}$. There is no rule stronger than $r_{1}$, and thus no conclusion can be defeasibly proven.

$+\partial$ : If $P(l+i, c+1)=+\partial \varphi$, then

(1) $+\Delta \varphi \in P(l, c)$ or

(2) (1) $-\Delta \sim \varphi \in P(l, c)$ and

(2) $\exists r \in R_{s d}[\varphi, j] \partial$-sequence-consumable and

(3) $\forall s \in R[\sim \varphi]$ either

(1) $s$ is $\partial$-sequence-discarded, or

(2) $\exists t \in R[\varphi] \partial$-sequence-consumable, $t \succ s$, and

(3) if $\exists w \in R[\sim \varphi] \partial$-sequence-applicable, $t \succ w$, then

(1) $\forall \alpha_{j} \in A(t), \alpha_{j}$ is $\partial$-consumed, otherwise

(2) $\forall \alpha_{k} \in A(r), \alpha_{k}$ is $\partial$-consumed.

Consider $D$ of Example 4, again with $C(r)=\{\varphi, \chi, \psi\}$. This time $r_{1}$ can prevent only to prove $+\partial \chi$ (and, analogously, $r_{0}$ prevents to conclude $+\partial \sim \chi$ ), and thus we prove $+\partial \varphi$ as well as $+\partial \psi$.

\section{Conclusions, related and further work}

We have dealt with the problem of manipulating resource consumption in non-monotonic reasoning. The combination of linear and defeasible features in a logical system is a complete novelty in the community of computational logic and knowledge representation.

Variants of SDL have been investigated so far as a means for devising business process traces [13 12[4]. While the idea is closely related to outline in the Introduction that a derivation corresponds to a trace in a process, the approach based on variants of SDL are not able to handle loops and, in general, repetitions of tasks. These aspects are elegantly captured by the sub-structural aspects presented in the paper.

Studies on light linear logic versions, with specific aspects of linearity related to resource consumption have been devised such as light and soft linear logic [35]. Applications of linear logic to problems indirectly related to business processes such as Petri Nets can be found in [9] and in [17/2]. However, such approaches are not able to handle in a natural fashion the aspect of exceptions. The representation of exception would require complex rules and encyclopaedic knowledge of the scenarios described by the processes encoded by rules/sequents.

The most important properties we aim at proving for a logical non-monotonic system are consistency and coherence4. Although we cannot report a formal proof of consistency and coherence for the logical system so far, we have exhaustively determined the conditions for interference of the sub-structural and the non-monotonic aspects of RSDL, and the formalisation of these derives the aforementioned properties.

A logical system enjoys the finite model property when for every set of formulae, the associated meaning to each formula requires a finite set of elements in the semantics for every model of that set. In the case of RSDL, the semantics is determined by the derivations that are possible given a theory.

\footnotetext{
${ }^{4}$ In DL, a theory $D$ is consistent if, for no literal $\varphi, D \vdash+\# \varphi$ and $D \vdash+\# \sim \varphi ; D$ is coherent if, for no literal $\varphi, D \vdash+\# \varphi$ and $D \vdash-\# \varphi$, \# $\in\{\Delta, \partial\}$.
} 
As a consequence of the aforementioned notions we shall prove one property that regards acyclic RSDL. The Atom Dependency Graph 5 of a defeasible theory has been defined in many different contexts, specifically in the analysis of preferences, as in [6]. Acyclic RSDLs are theories in which no cycle appears in the Atom Dependency Graph. This means that when a rule is used to produce a conclusion, the resources in the antecedent of the rules cannot be replenished, and we reach nodes, literals, that can be produced by the theory only if they are given (node, with no incoming edges in the Atom Dependency Graph).

Theorem 1. Acyclic finite RSDL theories enjoy the Finite Model Property.

Proof. If there are no cycles in the Atom Dependency Graph, every time a rule is used to derive a positive conclusion (strict or defeasible), the number of available resources decreases. Accordingly, the maximum number of literals that can appear in a proof $P$ is bounded and proportional to the number of literals occurring in the head of rules in a given theory. Consequently, every derivation is finite. Hence, the theory has the Finite Model Property.

Note that the theory with $\alpha$ as a fact and the rule $\alpha \Rightarrow \alpha$ can generate a derivation with infinitely many occurrences of $\alpha$. The acyclicity condition allows us to compute in finite time the extension of a theory. However, this is not the case for cyclic theory, where the computation is not guaranteed to terminate. Accordingly, we can state the following result.

Theorem 2. The problem of computing extensions of cyclic RSDL theories is semidecidable.

For acyclic theories, we have the finite model property. Therefore, since acyclic theories can be checked for model existence in finite time, when the model does not exists, and by brute force methods (for instance by simply computing all the possible sequences of any finite length) we can trivially claim the following result.

Theorem 3. The problem of computing extensions of acyclic RSDL theories is decidable.

Generally speaking, the extension computation problem is likely to be decidable for larger classes of pure acyclic theories. In particular, when cycles are conservative (cycles that do not produce new instances of the same literal), or when a cycles are limited in the extension by some rules that consume the literals generated in the cycles themselves before such literals could be used to allow other rules fire, it is possible to guarantee the finite model property. This is a matter of future investigations.

DL has been introduced as a means for managing non-monotonic aspects of logical conclusion/derivation mechanisms. DL is efficient in terms of time and space, being the problem of computing the extension of a defeasible theory linear in the number of literals in the theory. This property, however, cannot be claimed for RSDL. In particular,

\footnotetext{
${ }^{5}$ In the Atom Dependency Graph the atomic propositions are the nodes, and there is a directed edge between nodes if there is a rule containing the source or its negation in the body, and the target or its negation in the head.
} 
we can show that RSDL can be used to represent classical 3-SAT problem, and therefore prove that the complexity of this problem cannot be polynomial on deterministic machines.

The basic idea of reducing 3-SAT is as follows. A 3-SAT problem $P$ is a clause representing a finite conjunction of triplets $\left(t_{i}\right)$, each formed by three literals $\left(t_{i}^{1}, t_{i}^{2}, t_{i}^{3}\right)$, that we assume to be conjuncted in the sub-clause, where we ask whether the clause is satisfiable, or not.

We map each literal appearing in the clause in a positive literal $\widehat{t_{i}^{x}}$ (with $x=1,2,3$ ), not appearing in the clause, and add one positive literal $\widehat{t_{i}}$ for every triplet, again not appearing in the triplets. Subsequently, we add one rule $\widehat{t_{i}} \Rightarrow \widehat{t_{i}^{x}}$ for each of the three values $x=1,2,3$ and three rules $\widehat{t_{i}^{x}} \Rightarrow t_{i}^{x}$ for each of the values $x=1,2,3$. Finally, we add one fact for every literal $\widehat{t_{i}}$. Conclusively, we have mapped every triplet in six RSDL rules. The resulting RSDL theory has a derivation containing at least one literal for each clause if and only if the original problem $P$ is a satisfiable clause. For example, consider the clause $(\alpha \vee \beta \vee \gamma) \wedge(\neg \alpha \vee \neg \beta \vee \delta)$. Using $c_{1}$ and $c_{2}$ for the triplets, and $c_{1}^{1}, c_{1}^{2}, c_{1}^{3}, c_{2}^{1}, c_{2}^{2}, c_{2}^{3}$ for the elements in the triplets, the theory encoding the clause is:

$$
\begin{array}{ccccc}
c_{1} & c_{1} \Rightarrow c_{1}^{2} & c_{2} \Rightarrow c_{2}^{2} & c_{1}^{2} \Rightarrow \beta & c_{2}^{2} \Rightarrow \sim \beta \\
c_{2} & c_{1} \Rightarrow c_{1}^{3} & c_{2} \Rightarrow c_{2}^{3} & c_{1}^{3} \Rightarrow \gamma & c_{2}^{3} \Rightarrow \delta \\
c_{1} \Rightarrow c_{1}^{1} & c_{2} \Rightarrow c_{2}^{1} & c_{1}^{1} \Rightarrow \alpha & c_{2}^{1} \Rightarrow \sim \alpha &
\end{array}
$$

In this paper we presented the general idea of how to develop a logic combining features for sub-structural logic and defeasible reasoning, and we provided some general results about meta-theoretic properties (e.g., decidability and computational complexity). More research is required to determine the correct boundary between decidable and undecidable problems for these types of hybrid combinations and to provide a full map of the computational complexity analysis of the various options. However, the outline we discussed in this section seems to indicate that this is not a straightforward task. In this paper, we did not address the issue of how to model the motivational attitudes of the agents, we left the investigation of how to extend the framework to integrate with the framework of [8]7]. Related to this, we shall look at the problem of Business Process Compliance, in order to determine how to employ RSDL for marking up traces of processes corresponding to the execution of the a theory.

\section{References}

1. Antoniou, G., Billington, D., Governatori, G., Maher, M.J., Rock, A.: A family of defeasible reasoning logics and its implementation. In: Horn, W. (ed.) ECAI. pp. 459-463. IOS Press (2000)

2. Engberg, U., Winskel, G.: Completeness results for linear logic on petri nets. Ann. Pure Appl. Logic 86(2), 101-135 (1997)

3. Gaboardi, M., Marion, J.Y., Ronchi Della Rocca, S.: Soft linear logic and polynomial complexity classes. Electronic Notes in Theoretical Computer Science 205(C), 67-87 (2008)

4. Ghooshchi, N.G., van Beest, N., Governatori, G., Olivieri, F., Sattar, A.: Visualisation of compliant declarative business processes. In: EDOC 2017. pp. 89-94. IEEE Computer Society (2017)

5. Girard, J.Y.: Light linear logic. Information and Computation 143(2), 175-204 (1998)

6. Governatori, G., Olivieri, F., Scannapieco, S., Cristani, M.: Superiority based revision of defeasible theories. In: RuleML 2010. pp. 104-118. LNCS 6403, Springer (2010) 
7. Governatori, G., Olivieri, F., Scannapieco, S., Rotolo, A., Cristani, M.: The rational behind the concept of goal. Theory and Practice of Logic Programming 16(3), 296-324 (2016)

8. Governatori, G., Rotolo, A.: BIO logical agents: Norms, beliefs, intentions in defeasible logic. Journal of Autonomous Agents and Multi Agent Systems 17(1), 36-69 (2008)

9. Kanovich, M., Ito, T.: Temporal linear logic specifications for concurrent processes. In: LICS 1997

10. Küngas, P., Matskin, M.: Linear logic, partial deduction and cooperative problem solving. In: DALT II. pp. 263-279. LNCS 3476, Springer (2004)

11. Nute, D.: Defeasible logic. In: Handbook of Logic in Artificial Intelligence and Logic Programming, vol. 3. Oxford University Press (1987)

12. Olivieri, F., Cristani, M., Governatori, G.: Compliant business processes with exclusive choices from agent specification. In: PRIMA 2015. pp. 603-612. LNCS 9387, Springer (2015)

13. Olivieri, F., Governatori, G., Scannapieco, S., Cristani, M.: Compliant business process design by declarative specifications. In: PRIMA 2013. pp. 213-228. LNCS 8291, Springer (2013)

14. Pham, D.Q., Harland, J.: Temporal linear logic as a basis for flexible agent interactions. In: AAMAS '07. pp. 28:1-28:8. ACM (2007)

15. Pham, D.Q., Harland, J., Winikoff, M.: Modeling agents' choices in temporal linear logic. In: DALT V. pp. 140-157. LNCS 5397, Springer (2008)

16. Rao, J., Küngas, P., Matskin, M.: Composition of semantic web services using linear logic theorem proving. Information Systems 31(4-5), 340-360 (2006)

17. Tanabe, M.: Timed petri nets and temporal linear logic. In: ICATPN 1997. pp. 156-174. LNCS 1248 (1997) 